\title{
HACIA UNA REINTERPRETACIÓN DE LA CIENCIA NORMAL: KUHN Y LA FÍSICA DE SU TIEMPO (1940-1951)*
}

\section{Juan Vicente Mayoral de Lucas}

Universidad de Zaragoza

\begin{abstract}
RESUMEN
Este artículo habla de la experiencia de Thomas Kuhn como físico: su educación en la disciplina, su servicio como experto durante la Segunda Guerra Mundial, y su investigación doctoral. Estudiamos un lapso de tiempo que va de 1940 a 1951. El objetivo en última instancia es ofrecer base empírica para valorar una afirmación como la de Mara Beller (1999), que dice que la descripción kuhniana de la ciencia normal está parcialmente basada en circunstancias y experiencias personales, no sólo en los datos históricos o en la evidencia disponible de la sociología de la ciencia. En este artículo se establece que dicha tesis es plausible.
\end{abstract}

PALABRAS CLAVE: Educación progresista. Física del estado sólido. Segunda Guerra Mundial. Radar. Contramedidas. Teoría de bandas. WKB. Defecto cuántico. Thomas Kuhn. Mara Beller. John Van Vleck. Eugene Wigner. Frederick Seitz.

\section{TOWARDS A REINTERPRETATION OF NORMAL SCIENCE: KUHN AND THE PHYSICS OF HIS TIME (1940-1951)}

\section{ABSTRACT}

This paper deals with Thomas Kuhn's experience as a physicist — his training in the field, his brief period as a physicist during World War II, and his doctoral research — from 1940 to 1951. Its aim is to offer a basis to assess statements as Mara Beller's (1999) that Kuhn's account of normal science is partially founded on personal circumstances and experience, and not only on the historical record or available evidence from the sociology of science - a thesis here considered highly credible.

* Agradezco a Philip Anderson, Javier García Sanz, Yolanda García, Karl Hufbauer, Pierre Noyes, Manuel Sellés, Carlos Solís y a los revisores anónimos de la revista Asclepio su colaboración en la preparación y mejora del presente artículo. 
KEY WORDS: Progressive education. Solid-state physics. World War II. Radar. Jamming. Band theory. WKB. Quantum defect. Thomas Kuhn. Mara Beller. John Van Vleck. Wigner. Seitz.

\section{INTRODUCCIÓN}

No se suelen manejar demasiados datos acerca del periodo que Thomas Kuhn pasó estudiando e investigando como físico. Es un periodo relativamente corto dentro de su trayectoria. Dio comienzo en 1940, cuando entró en la Universidad de Harvard como estudiante, y acabó poco más de una década después, en 1951, aproximadamente cuando, ya defendida su tesis doctoral, publicó el último artículo extraído de ella (aunque ya desde 1949 no se dedicaba de lleno a la disciplina). Por otro lado, su filosofía de la ciencia nace principalmente de sus reflexiones sobre la historia de la ciencia más que sobre la ciencia contemporánea a él, con lo que parece que prescindir de los detalles de esa fase no supone perjuicio alguno para nuestro conocimiento de su obra.

La convicción que subyace a este artículo es que conocer un poco mejor ese periodo puede ofrecer algunos beneficios. El primero de ellos, aunque menor, no es insignificante: lograremos evitar esos errores de puro dato biográfico en que algunos autores han incurrido en ocasiones ${ }^{1}$. El segundo beneficio es más significativo: averiguar el origen extra-historiográfico del concepto kuhniano de «ciencia normal». Como es bien sabido, éste es uno de los conceptos claves de Kuhn, principalmente expuesto en 1962 en The Structure of Scientific Revolutions y explorado en mayor profundidad en The Essential Tension $^{2}$. En este segundo volumen, Kuhn habla de su importancia para el desarrollo científico y menciona algo significativo de pasada:

1 Cf., e.g., SARdar, Z. (2000), Thomas Kuhn and the Science Wars, Londres, Icon, p. 24, donde se indica que Kuhn hizo su doctorado bajo la supervisión de P.W. Bridgman, en lugar de J.H. Van Vleck. Incluso filosóficamente, la supervisión de Bridgman pudiera haber cambiado las cosas en la trayectoria posterior de Kuhn. Para más detalles sobre la tesis doctoral de Kuhn, cf. más abajo, quinta sección del artículo.

2 Kunn, T.S. (1962), The Structure of Scientific Revolutions, Chicago, The University of Chicago Press; Kunn, T.S. (1977), The Essential Tension, Chicago, The University of Chicago Press. Kuhn confiesa en el prefacio (p. xviii) a The Essential Tension que el artículo que da nombre al volumen (The Essential Tension: Tradition and Innovation in Scientific Research) contiene «un modesto desarrollo de la noción de ciencia normal». Otros ensayos de ese mismo volumen ahondan también en dicho concepto. 
Algo de divergencia caracteriza todo trabajo científico [...]. Pero mi propia experiencia en la investigación científica y mi versión de la historia de las ciencias me lleva a preguntarme si la flexibilidad y la apertura de mente no han sido subrayadas de manera demasiado exclusiva como las características requeridas para la investigación básica ${ }^{3}$.

Frente a tal flexibilidad y apertura de mente, Kuhn contrapone, como sabemos, un tipo de pensamiento menos crítico, más dogmático y tradicionalista, propio de su ciencia normal. Lo más importante del párrafo, no obstante, es que Kuhn señala que una de las fuentes de esa visión es su propia experiencia como físico. Puesto que su exposición de la ciencia normal es uno de los aspectos más sugerentes y (en la década de 1960) novedosos de su obra, comprobar su origen biográfico y averiguar hasta qué punto puede haberse construido sobre él podría ser revelador para nuestra comprensión de esa obra. En este artículo obtendremos algunos resultados de relevancia para satisfacer este objetivo.

Hay un tercer beneficio de esta clase de investigación biográfica, relacionado con el anterior, aunque éste es principalmente para los críticos de la concepción kuhniana de la ciencia normal. El trabajo de la historiadora Mara Beller lo ilustra con claridad. Beller se opone al dogmatismo inherente a la concepción de la ciencia normal de Kuhn. Ya hace años avisó de que la potencial conexión entre Structure y el periodo de Kuhn como físico podría explicar el excesivo énfasis kuhniano en la naturaleza dogmática de la ciencia normal. Esto queda patente en el siguiente extracto de su Quantum Dialogue:

La creatividad y longevidad de lo que Kuhn llamó «ciencia normal» se deben, sugiero, no a su dogmatismo sino a su carencia de él. La resistencia de la ciencia normal es posible porque no encaja bien en la noción kuhniana de ciencia normal. $[\ldots]$

La noción de Kuhn del científico como alguien que resuelve rompecabezas parece especialmente apropiada como descripción de la experiencia del estudiante graduado y posgraduado de ciencias, tal como el propio Kuhn fue antes de pasarse a la historia de la ciencia ${ }^{4}$. Quizá la experiencia de un científico creativo maduro (no de un científico de una ciencia «madura») quede mejor caracterizada por David

3 KuHn (1977), p. 226. Cursivas mías. Al principio de ese ensayo (The Essential Tension), Kuhn se refiere a sí mismo, en tercera persona, como «the physicist-historian» (cf. p. 225).

4 Beller menciona en este punto, en nota al pie, el mismo texto de The Essential Tension (Kuhn (1977), p. 226) que hemos reproducido unos párrafos atrás. 
Finkelstein: «En el presente, la ciencia funciona con muchos de sus practicantes buscando y, a la vez, dudando de la existencia de lo que buscan $»^{5}$.

En la primera parte del texto reproducido, Beller resume su postura antikuhniana en lo que respecta a la noción de ciencia normal y su conexión con el dogma. En el segundo párrafo se deja ver que la ciencia normal de Kuhn es dogmática debido a que el propio Kuhn se educó como físico hasta un grado de especialización en el que la ciencia normal también es dogmática. Pasos más allá de esa fase del científico novel, indica Beller, ese dogmatismo se diluye y la crítica adquiere mayor importancia. En este artículo no vamos a examinar la idea de Beller y no vamos a criticar (ni a defender) el dogmatismo aparentemente inherente a la ciencia normal. Sólo vamos a examinar la base empírica para hacer la afirmación mencionada: la ciencia normal de Kuhn depende en buena medida de la experiencia de Kuhn como físico. Sí cabe decir, no obstante, que, en caso de que esta afirmación sea verosímil, como creemos que es, quizá la ciencia normal de Kuhn ofrezca un perfil sólo (y quizá demasiado) parcial de la imagen real de la ciencia. El resultado de este trabajo podría ser, así, beneficioso para la crítica de la ciencia normal de Kuhn. En cualquier caso, abre una vía de reinterpretación.

Para realizar nuestro examen, procederemos del modo siguiente. En la segunda sección mostraremos el trasfondo educativo del joven Kuhn (aquél que entra en Harvard en 1940 decidido a convertirse en físico teórico). Veremos que es un Kuhn que valora de forma especial la creatividad como elemento inherente a toda forma de actividad intelectual (incluyendo la ciencia). La sección siguiente muestra a Kuhn en sus primeros estudios de grado en Harvard, lo cual no supone ningún choque con respecto a las expectativas iniciales, pues el problema de física es, para este Kuhn, un simple reto intelectual. Las secciones cuarta y quinta sí encarnan un cambio de circunstancias, cuyo impacto en Kuhn se aprecia mejor por contraste con el escenario visitado en las dos secciones previas. Estas nuevas secciones hablan del trabajo de Kuhn como experto en ondas durante la Segunda Guerra Mundial y su investigación de doctorado (claramente «convergente») bajo la supervisión de John Van Vleck. La rutina inherente a esas prácticas causa el creciente hastío de Kuhn hacia la práctica de las ciencias. Como se deja ver en el artículo, la rutina en

5 Beller, M. (1999), Quantum Dialogue: The Making of a Revolution, Chicago, The University of Chicago Press, p. 306. Las cursivas son de Beller. La cita es de FinKELSTEIN, D. (1987), All Is Flux. En HiLey, B.T. y PeAt, F.D. (eds.), Quantum Implications: Essays in Honor of David Bohm, Nueva York, Routledge, pp. 289-294, esp. p. 291. 
que por entonces se ve inmerso no excluye una fuerte dosis de creatividad, pero ésta va asociada a un proyecto de investigación cerrado, que no busca más que mejorar las capacidades de cálculo de una teoría, la cual no se somete a crítica en sus fundamentos. Si buscamos una buena imagen de la ciencia normal ajena a la teoría de Kuhn, ésta se puede encontrar fácilmente aquí. Cerraremos el artículo con algunas reflexiones sobre lo visto hasta el momento y con un Apéndice que aporta datos relevantes para la sección quinta.

\section{EL JOVEN TOM KUHN}

Kuhn nació en Ohio en 1922, pero creció en Nueva York. Sus padres, Minette Stroock Kuhn y Samuel Kuhn, le procuraron, tanto a él como a su hermano menor, Roger, una educación liberal, «progresista», como se conocía por entonces. El matiz es significativo, porque la educación progresista huía de la escuela tradicional victoriana, del aprendizaje memorístico de las lecciones y de los castigos corporales, e introducía toda una serie de reformas encaminadas a la preparación del individuo para enfrentarse de manera eficiente y creativa a su propio ambiente. La escuela progresista seguía las directrices de John Dewey, quien advertía de que el aprendizaje del individuo no es un mero registro acumulativo de hechos monolíticos con los que equipar una mente vacía ${ }^{6}$. El ser humano corrige poco a poco una imagen inicial simplista que nace de su interacción con su entorno vital, con lo que educar al individuo conlleva partir del mismo punto de vista que éste ya lleva consigo a las aulas, especificarlo y ampliarlo, sin imponer modificaciones en él que no nazcan del propio juicio individual. Es decir, no sólo se trata de enseñar a sumar, sino de mostrar qué relaciones tienen las operaciones aritméticas con el mundo que a uno le rodea, desde su uso en la tecnología de la época a sus relaciones con formas más elevadas de pensamiento simbólico. Así, esa actividad adquiere sentido en el modo de vida del individuo ${ }^{7}$. En la Lincoln School de Nueva York, donde Kuhn estudió hasta los once años, y al igual

6 Cf. ReEse, W.J. (2001), The Origins of Progressive Education, History of Education Quarterly, 41, pp. 1-24, esp. pp. 10-11; DEwEY, J. (1896), The Reflex Arc Concept in Psychology, Psychological Review, 3, pp. 357-70; Dewey, J. (1899), The School and Society, Chicago, The University of Chicago Press; Dewey, J. (1916), Democracy and Education, Nueva York, Macmillan.

7 Cf. Cremin, L.A. (1961), The Transformation of the School, Nueva York, Alfred A. Knopf, esp. pp. 282-285. 
que en la Hessian Hills de Croton-on-Hudson, estado de Nueva York, donde estuvo hasta los quince, hablar de álgebra superaba en importancia a ejecutar mecánicamente meras divisiones y resolver ecuaciones de segundo grado. La interpretación histórica, sociológica y económica de los orígenes de la Constitución de los Estados Unidos de Charles A. Beard era, ya para estos jóvenes, tema de vivo debate ${ }^{8}$.

Las universidades de la Ivy League tal vez clamaran por individuos así educados, pero el acceso a ellas a través de la escuela preparatoria seguía siendo sinónimo de tradicionalismo ${ }^{9}$. Por eso, Kuhn, que pretendía ir a Harvard como las generaciones previas de su familia, debía asistir a alguna escuela tradicional donde cultivar de manera rigurosa y promocionar sus diversos talentos: desde la discusión en los foros académicos a sus notables capacidades para el cálculo y el pensamiento abstracto. Así, en las escuelas preparatorias (Solebury, en Pennsylvania, y Taft School, en Connecticut) Kuhn pasó unos años mucho más rigurosos y duros que los precedentes.

En general, Kuhn no parece haber sido un muchacho terriblemente popular, pero sí un habilidoso orador que ya en 1936 pronunció algún discurso a favor del pacifismo ${ }^{10}$. Era un joven competitivo, según se manifiesta en su registro de la Taft, cuyo interés por los deportes le llevó en alguna ocasión a alguna pelea poco antes de salir para Harvard. Acabó la educación secundaria Cum Laude, perteneció a varios grupos de debate y deportes y ganó premios como la Rensselaer Alumni Association Medal por un trabajo excepcional en matemáticas y ciencias ${ }^{11}$. Era un joven de éxito. Aunque los años le harían calmar su furor, siempre mantuvo esa vena de independencia, libertad de pensamiento y racionalismo aprendida en familia y en las escuelas progresistas a

8 Baltas, A., Gavroglu, K., Kindi, V. y Kuhn, T.S. (2000), A Discussion with Thomas S. Kuhn. En Kunn (2000), The Road since Structure, Conant, J. y Haugeland, J. (eds.), Chicago, The University of Chicago Press, pp. 256-323, esp. pp. 256-257.

9 Cf. Saveth, E.N. (1988), Education of an Elite, History of Education Quarterly, 28, pp. 367-386.

10 Kunn, T.S. (1936), Speech on Day of Student Strike. En Thomas S. Kuhn Papers, 1922-1996, MC 240, Institute Archives and Special Collections, Massachusetts Institute of Technology, Cambridge, Mass., caja 1, carpeta 2. En adelante, estos archivos se mencionarán mediante la abreviatura TSKP, seguido de un número que señala la localización del documento en los mismos, que, en el caso anterior, hubiera sido «1.2».

11 Hufbauer, K.F. (1997), «Kuhn's Discovery of History (1940-1958)». MS, Apéndice A. Agradezco a Karl Hufbauer su amabilidad al proporcionarme un borrador de este magnífico artículo, lamentablemente aún sin publicar. Varios (yo diría que todos los) investigadores de la biografía de Kuhn nos hemos beneficiado de este texto pionero. 
las que asistió. En 1941 afirmaba que la base de todas sus ideas era una sólida fe en la razón como único medio para ofrecer argumentos válidos y, de ese modo, resolver problemas. El ser humano, asumía ya entonces, siempre acaba por someterse al dictado de la razón ${ }^{12}$.

\section{KUHN EN HARVARD: EL APRENDIZAJE DE UNA CIENCIA}

En los Estados Unidos de la década de 1940, las perspectivas laborales para un joven con un título de física por la Universidad de Harvard eran mejores que las de un matemático ${ }^{13}$. El padre de Kuhn, Samuel L. Kuhn, un asesor financiero de Nueva York, ingeniero y alumno de Harvard y el MIT, tenía esto bastante claro y así se lo hizo saber a su primogénito. A su padre no le faltaba razón. La física había cambiado en Estados Unidos desde que se cultivase con objetivos principalmente prácticos (la industria de la comunicación, los recursos energéticos, la agricultura, etc.). Como ha mostrado Daniel Kev$\operatorname{les}^{14}$, desde comienzos del siglo XX, la industria privada y el gobierno norteamericanos habían buscado emanciparse de los laboratorios europeos tanto por el ahorro económico como por el mero prestigio. American Telegraph and Telephone (AT\&T), General Electric o el National Bureau of Standards querían que la física norteamericana controlase y desarrollase por sí misma la base teórica de la disciplina, una autonomía que se volvió necesaria con el bloqueo británico durante la Primera Guerra Mundial. La competencia europea al final de la contienda disparó del todo estos planes norteamericanos. En 1924, la AT\&T fundó los Bell Laboratories, que se convirtieron en un foco de investigación básica de primer orden, y el gobierno destinó instalaciones propias, como el Naval Research Laboratory, a ese mismo objetivo. Así, cuando Kuhn accedió a la universidad, un físico joven podía crecer en su profesión si

12 Kunn, T.S. (1941), The War and My Crisis. En TSKP, 1.3, esp. pp. 2-3. Para más detalles sobre ese ensayo inédito de Kuhn, cf. AndresEN, J. (1999), Crisis and Kuhn, Isis, 90 (suplemento), S43-S67; MAYORAL, J.V. (2009), Intensions, Belief and Science: Kuhn's Early Philosophical Outlook (1940-1945), Studies in History and Philosophy of Science, 40, pp. 175-184.

13 Sigurdsson, S. (1990), The Nature of Scientific Knowledge: An Interview with Thomas Kuhn, Harvard Science Review, 3, pp. 18-25, esp. p. 18.

14 Kevles, D. (1988), Physics and National Power, 1870-1930. En GoldberG, S. y Stuewer, R.H. (eds.), The Michelson Era in American Science, 1870-1930, Nueva York, American Institute of Physics, pp. 248-257; Kevles, D. (1995), The Physicists, Cambridge, Mass., Harvard University Press. 
tenía el talento suficiente y accedía o a los ya abarrotados laboratorios universitarios, o a los privados y gubernamentales, donde la investigación era más corporativa que en las universidades, pero los presupuestos acumulaban una gran cantidad de ceros. Aunque él prefiriese estudiar matemáticas, los argumentos de su padre eran muy convincentes, con lo que, al entrar en Harvard, Kuhn ya tenía clara su elección de carrera universitaria: la física teórica.

La física norteamericana de aquella época no era, sin embargo, el ámbito adecuado para desarrollar una carrera marcada por la creatividad y la independencia intelectual. Algo así se dejaba para la literatura (que Kuhn continuó estudiando y practicando en Harvard cuando el resto de asignaturas se lo permitía $)^{15}$. Ya en las escuelas preparatorias, por las que Kuhn, recordemos, había pasado, se subrayaba el dominio estricto de la física clásica y las universidades privilegiaban, para sus carreras técnicas y científicas, la calidad del alumnado entrante por encima de la cantidad. En especial, valoraban la destreza matemática ${ }^{16}$. El propio Kuhn aún recordaba bastante bien pasadas varias décadas que su profesor de física y química en la Taft, Sidney Hadley, hacía muy poco caso a sus intentos de desarrollar puntos de vista independientes en física ${ }^{17}$.

Con todo, Kuhn entró en Harvard en el otoño de 1940 decidido a estudiar física. Las perspectivas profesionales eran, como decimos, suficientemente convincentes, pero además Kuhn tenía un gran «interés en problemas fundamentales» y la física parecía poder satisfacerlos ${ }^{18}$. Por ello, Kuhn no tuvo ninguna duda desde un principio acerca de las asignaturas que debía elegir. El estudiante de primer año de licenciatura en Harvard tenía que decidir su carrera al final del semestre. Antes de llegar a ese punto, se le recomendaba diversificar las cuatro o cinco asignaturas correspondientes y dedicarse a varias cosas que no tuviesen nada que ver con la carrera preferida, para así evitar arrepentimientos tardíos. Si exceptuamos Mathematics A (geometría analítica y cálculo infinitesimal), obligatoria para los futuros estudiantes de física, y la inevitable asignatura de inglés (excepto para los que entraban con mención de honor en la materia), restan un par de asignaturas, tres en el mejor de los casos, que dedicar a otras disciplinas. Kuhn no tuvo demasiadas dudas ni

15 Cf. MAYORAL (2009), §2.

16 Cf. Donahue, D.M. (1993), Serving Students, Science, or Society? The Secondary School Physics Curriculum in the United States, 1930-65, History of Education Quarterly, 33, pp. 321-52.

17 Baltas et al. (2000), p. 258; Hufbauer (1997), Ap. A; Sigurdsson (1990), p. 18.

18 BALTAS et al. (2000), p. 258; SigURDSSON (1990), pp. 18-19. 
diversificó demasiado. Se matriculó en Physics $F$, que duraba dos años y tenía fama de ser bastante complicada, y en una asignatura de historia de la filosofía con Raphael Demos que le marcaría sobremanera ${ }^{19}$. Sus restantes intereses, principalmente literatura y política, reaparecerían en su expediente periódicamente, pero sobre todo los cultivaría como parte de su actividad extra-académica, considerablemente rica en su caso, pues perteneció a diversos grupos de debate literario y político, y acabó dirigiendo el consejo editorial del prestigioso diario estudiantil The Crimson ${ }^{20}$.

Las asignaturas de matemáticas no fueron un problema para Kuhn hasta el tercer año de carrera. Kuhn se sentía muy por encima de la media. El primer año dejó de asistir a clase después de pocas semanas; solía resolver los problemas asignados por sí solo y enviarlos con algún compañero. Sólo el segundo semestre del segundo año le empezó a revelar cosas nuevas, aunque los problemas realmente serios empezaron a aparecer con la asignatura de tercero que impartía el famoso George Birkhoff. Las integrales múltiples y la diferenciación parcial se le revelaron difíciles y años más tarde confesaba que incluso después no siempre lograba resolver las primeras ${ }^{21}$. Por otro lado, Kuhn tampoco recuerda que sus primeros contactos con la física universitaria fuesen del todo buenos, de acuerdo con sus propias impresiones posteriores. Aparentemente, la causa de ello fue la necesidad de subir un escalón de dificultad notablemente marcado. Physics $F$, la asignatura elegida por él ya en primero, comenzaba siendo impartida en el primer año por Wendell Furry, un antiguo colaborador de J.R. Oppenheimer en Berkeley (en lo que había constituido una de las primeras ventanas del país al nuevo paisaje de la cuántica

19 Sobre estas asignaturas, cf. The Crimson Confidential Guide to Freshman Courses 1940 (1940), Cambridge, Mass., The Harvard Crimson, pp. 19-20; Harvard University Archives, Harvard University, Cambridge, Massachusetts, Ref.: HUD 3304 240. En adelante, los archivos de Harvard se abreviarán como «HUA». Sobre el aprendizaje de Kuhn con Demos, cf. MAYORAL (2009), §2.

20 BALTAS et al. (2000), pp. 264-265 y 268. Muchos de estos detalles aparecen reflejados en diferentes documentos localizados en los archivos de Harvard y el MIT. Un breve listado de las actividades extra-académicas de Kuhn aparece en Class of 1944 Freshman Red Book, p. 105, HUA, Ref.: HUD 348. La actividad de Kuhn en The Crimson se puede consultar en los diversos volúmenes del Crimson Editorial Comments Book (HUA, Ref.: HUD 3304.721), en especial, a partir de 1942. Los trabajos de estudiante de Kuhn aparecen en TSKP 1.3. Para un estudio sobre algunos de los aspectos relevantes de dichos trabajos para su pensamiento filosófico, cf. MAYORAL (2009).

21 BALtAS et al. (2000), p. 267. 
europea $)^{22}$. Furry tenía un nivel teórico bastante alto y Kuhn no parece haberse sentido al principio capaz de seguir su ritmo. Kuhn recordaba no saber cómo enfrentarse bien a los problemas (principalmente de mecánica, calor y sonido) propuestos por Furry. Sus calificaciones empezaron a bajar (algo inaudito en Kuhn, un alumno tradicionalmente sobresaliente) y tuvo sus primeras dudas sobre su talento para esta materia. Kuhn logró resolver el problema, aunque el reto parece haberle dejado huella. La superación de la dificultad provino, según su propia versión, de algo parecido a un «momento de iluminación» en el que aprendió de repente el modo específico de resolver esos problemas ${ }^{23}$. Para él, esta sensación sería clave en el futuro, cuando por ejemplo distinguiera el patrón de práctica científica propia de la ciencia normal en Structure como una tradición de solución de rompecabezas, ya que el aprendizaje de esa clase de práctica, la entrada en dicha tradición, suele acarrear parecidas experiencias de comprensión repentina de un modo de describir fenómenos y resolver problemas ${ }^{24}$.

Tuviera algunas dificultades o no, la impresión general que producía Kuhn, entre sus éxitos académicos y sus ricas actividades extra-académicas, era la de un prometedor joven de gran talento. De hecho, se graduaría Summa Cum Laude un año antes que su promoción ${ }^{25}$ y entraría directamente a formar parte de uno de los proyectos de investigación en física desarrollados por el Gobierno de Estados Unidos durante la guerra: el desarrollo de contramedidas de radar (cf. nuestra siguiente sección). Philip Anderson, compañero suyo de estudios (y futuro premio Nobel de Física) recordaba recientemente, con notable modestia por su parte, al Kuhn de aquella época del modo siguiente:

PHILIP ANDERSON: [...] yo no era un prodigio académico como Tom Kuhn [...].

ALEXEI KoJeVNiKOV: ¿Era Kuhn un prodigio académico en aquella época?

ANDERSON: Oh, sí. Lo era todo. Era el gran hombre del campus.

KOJEVNIKOV: ¿Estaba en tu clase?

22 Sobre Furry y ese contexto, cf. SchweBER, S.S. (1986), The Empiricist Temper Regnant: Theoretical Physics in the United States, 1920-1950, Historical Studies in the Physical Sciences, 17, pp. 55-98, esp. pp. 88-89; Brown, L.M., PAIS, A. y PIPPARD, B. (1995), Twentieth Century Physics, Bristol, Institute of Physics Publishing, vol. 2, pp. 640-41.

23 Cf. BALTAS et al. (2000), pp. 261-62 y 267.

24 Cf., e.g., KUHN (1962), pp. 36-39; KUHN (1977), p. 305; KUHN (2000), p. 178.

25 Algo que sólo 500 de 2000 alumnos lograron hacer (Comunicación personal con H.P. Noyes, 11 de enero de 2003. Noyes, a quien agradezco su colaboración, fue compañero de estudios de Kuhn y más tarde se lo volvió a encontrar en Berkeley, donde Kuhn trabajaría, ya como historiador de la ciencia, años después). 
ANDERSON: Sí, en la misma clase. Era editor del periódico The Crimson. Era junior marshal Phi Beta [Kappa], fue nombrado para Phi Beta cuando era junior. Nunca obtuvo otra cosa que no fuera un sobresaliente. Siempre que estaba en la misma clase que él, procuraba estar un punto o dos por encima de su nivel, pero yo consideraba que aquello era lo máximo a lo que yo podía aspirar ${ }^{26}$.

Pese a lo dicho, el «gran hombre del campus» se enfrentaría a problemas que poco a poco minarían su tendencia hacia la física teórica. Kuhn mantuvo su independencia intelectual y ahondó en ella, pero pronto se dio cuenta de que convertirse en físico profesional exigiría de él un menor cultivo de la misma. Por otro lado, como dijimos, ya en su primer año en Harvard había coqueteado con la filosofía, y esa experiencia le dejó huella. A finales de julio de 1943, nada más graduarse, escribía a su tía Emma Fisher que sus inclinaciones intelectuales tenían más que ver con las reflexiones filosóficas acerca de la física que con la física misma ${ }^{27}$. La guerra, no obstante, había desviado su trayectoria hacia ésta última. Kuhn dedicaría oficialmente la mayor parte de su tiempo y esfuerzo a la física hasta finales de 1948, la fecha en que obtendría su doctorado. Algo que le quedaría bastante claro a lo largo de los cinco años siguientes (1943-48) sería que la física exigía limitar la atención a un aspecto específico, restringido, de la realidad (si ésta era una falsa impresión o no es ya otra cuestión ${ }^{28}$ ). Esta experiencia, relatada en las dos secciones siguientes, influyó definitivamente en su abandono de la física, aunque también, defendemos aquí, en la imagen de la ciencia que contribuyó a extender.

\section{KuHn DuRante La Segunda Guerra Mundial}

\subsection{Kuhn, Harvard y las contramedidas}

El 22 de diciembre de 1941, la marina norteamericana propuso un programa para el uso en el campo de batalla de contramedidas de radar que el Na-

26 Entrevista de A. Kojevnikov a P.W. Anderson, 30 de marzo de 1999, Niels Bohr Library \& Archives, American Institute of Physics, College Park, Maryland, EE.UU. (www.aip.org/history/ohlist/23362_1.html).

27 Carta de Kuhn a E.K. Fisher, 27 de julio de 1943. TSKP, 12.33, pp. 2-3.

28 El propio Kuhn creía, décadas después (en los años noventa), que la impresión que tenía de la física al acabar la guerra estaba equivocada; en otras circunstancias, si, e.g., hubiera trabajado en Los Álamos, quizá hubiese continuado con su carrera de físico. Cf. BALTAS et al. (2000), p. 271; SIGURDSSON (1990), p. 19. 
tional Defense Research Committee (NDRC) debía desarrollar. El objetivo, en términos técnicos, consistía en especificar las características de los equipos alemanes de radar y desarrollar modos de anular sus efectos. Esta tarea debía ser llevada a cabo en colaboración con el Radiation Laboratory del MIT, pero este laboratorio tenía, ya por entonces, demasiadas competencias asignadas. Luis Alvarez ya había dedicado un grupo al desarrollo de contramedidas y esa experiencia debía ser aprovechada, aunque en otro lugar, preferiblemente cercano a Cambridge, Massachusetts ${ }^{29}$. Harvard era un emplazamiento apropiado. La División D del NDRC y esa universidad firmaron un acuerdo para instalar en su campus el laboratorio de contramedidas: el conocido como Radio Research Laboratory ${ }^{30}$. El 11 de febrero de 1942 (por recomendación de Álvarez), Frederick E. Terman, antiguo director del Departamento de Ingeniería Eléctrica de la Universidad de Stanford, fue nombrado director y éste reclutó a su vez a Roger Hickman, del propio Harvard, y a un par de personas más, para abrir las puertas del centro ${ }^{31}$. Para enero de 1944, cuando Kuhn ya se encontraba entre sus filas, el laboratorio contaba con 744 empleados, 214 de los cuales eran personal de investigación. El laboratorio, al principio dependiente del Radiation Lab del MIT, se emancipó de éste en marzo de 1942 y pasó de los edificios del MIT a un reformado bloque de biología de Harvard en Divinity Avenue ${ }^{32}$.

El laboratorio era oficialmente secreto para la población universitaria ${ }^{33}$, pero, tanto a él como a muchos otros estudiantes de física, se les animó a in-

29 Carta de Karl T. Compton a James B. Conant, 30 de enero de 1942, en Papers of James B. Conant, HUA, Ref.: UAI 15.898, caja 12, carpeta OSRD-NDRC 1942-1944.

30 No sería el único. Harvard instaló otros dos laboratorios para desarrollos tecnológicos útiles en la guerra: El Computer Laboratory y el Underwater Sound Laboratory. Cf. WELCH, G.W. y CoHen, A.R. (1999), Aiken's Program in a Harvard Setting. En CoHEn, I.B., Welch, G.W. y CAMPBell, R.V.D. (eds.), Makin' Numbers, Cambridge, Mass., The MIT Press, pp. 163-181, esp. p. 165.

31 Sobre Terman, cf. Galison, P. (1997), Image and Logic, Chicago, The University of Chicago Press, pp. 273-281; GeIGER, R.L. (1992), Science, Universities, and National Defense, 1945-1970. En Thackray, A. (ed.), Science After '40, Osiris, 7, pp. 26-48, esp. pp. 33-34.

32 Sobre todo ello, cf. BALTAS et al. (2000), pp. 268-69; BROwn, L. (1999), A Radar History of World War II, Bristol, Institute of Physics Publishing, p. 293; BUDERI, R. (1996), The Invention that Changed the World, Nueva York, Simon \& Schuster, p. 207; GuERLAC, H. (1987), Radar in World War II, Nueva York, American Institute of Physics, pp. 287 y 300.

33 Cf. Kunn, T.S. (1945a), Science at War, Harvard Alumni Bulletin, 48. Este texto no aparece firmado por Kuhn ni se encuentra en su lista de publicaciones, pero hay dos recortes del mismo en una carpeta de sus archivos titulada «Papers 1945-1948» (TSKP, 1.4) que sólo contiene sus propias contribuciones. Para Karl Hufbauer ([1997], nota 27), esto y el propio estilo del artículo sugieren que se trata de un texto redactado por el propio Kuhn, algo que no 
clinar sus pasos hacia la electrónica ${ }^{34}$. Esto lo alejó de una formación teórica más pura. Kuhn debería haber estudiado alguna asignatura de óptica, termodinámica (algo que dudaba haber tocado hasta el doctorado) y relatividad, pero estas materias sólo aparecieron en su expediente como parte de su formación doctoral. En su lugar, siguió un itinerario de estudios principalmente centrado en teoría electromagnética y electrónica con miembros del llamado «Cruft group» ${ }^{35}$. El «Cruft group» era un grupo de físicos especializado en la tecnología de la comunicación que se concentraron en torno al Cruft Laboratory de Harvard ${ }^{36}$. Su cabeza visible era el renombrado E.L. Chaffee, un especialista en electrónica y tubos de vacío y también un reconocido administrador con sólidos principios tradicionales. El Cruft, con Chaffee a la cabeza, puso sus conocimientos de electrónica a las órdenes de Conant, el rector de Harvard (y uno de sus destinos fue la instrucción en tecnología de radares para el ejército) ${ }^{37}$. Kuhn cursó asignaturas sobre, e.g., tubos de vacío (fenómenos de emisión y de descarga en gas) y teoría de circuitos con el propio Chaffee, y sobre electromagnetismo y teoría de antenas con R.P.W. King (un especialista en esta última materia) ${ }^{38}$. Con esta formación y una buena finalización de estudios, Kuhn fue directamente a trabajar al Radio Research Laboratory.

Allí, Kuhn seguiría en contacto con King, pero entraría a formar parte de un grupo teórico supervisado por quien sería su futuro director de tesis, John H. Van Vleck (de quien hablaré más abajo) ${ }^{39}$. La labor de Kuhn era hacer cálculos de la potencia estimada de los ecos de radar que devolverían los objetivos militares propuestos. Gracias a estas estimaciones, en las que se tenían en cuenta diferentes variables, desde la distancia hasta las condiciones medioambientales, y en las que se empleaban los datos proporcionados por el Naval Research Laboratory, se valoraba la efectividad de las contramedidas

sería raro dadas tanto su participación como redactor-jefe en el periódico estudiantil The Crimson durante sus estudios de licenciatura como otras colaboraciones puntuales para el Harvard Alumni Bulletin.

34 Así lo afirma Philip Anderson en su autobiografía. En: www.nobelprize.org/nobel pzizes/physics/laureates/1977/anderson-autobio.html (consultado el 25 de febrero de 2011).

35 BALTAS et al. (2000), pp. 267-68.

36 Cf. Cohen, I.B. (1999), Howard Aiken, Cambridge, Mass., The MIT Press, pp. 21-31.

37 Carta de James B. Conant a Vannevar Bush, 2 de junio de 1941, James B. Conant Papers, HUA, caja 12, carpeta OSRD-NDRC. NDRC-1940-1941, p. 4.

38 Kunn (1955), Vita. En Thomas S. Kuhn Dossier, depositado en The Bancroft Library, University of California, Berkeley; cf. también BALTAS et al. (2000), pp. 268-69.

39 Cf. Baltas et al. (2000), p. 269; KUHN (s.f. [a]), Preliminary Considerations Concerning Radar Echoes and RCM Parameters in Naval Operations. En TSKP, 1.8. 
desarrolladas en el Radio Research Laboratory. Así, por ejemplo, Kuhn pasó algún tiempo trabajando en las contramedidas apropiadas para la cobertura de radar japonés en Kamchatka ${ }^{40}$.

¿Qué era una contramedida? En resumen, un falso eco de radar. Su resultado era generar un esquema falso de la disposición de los bombarderos en un ataque aéreo. Uno de estos ingenios era, por ejemplo, «Window». (En Estados Unidos se denominaba «Chaff»). «Window» era adecuado para un tipo de radar alemán denominado «Würzburg», que operaba en una longitud de onda considerablemente corta, unos $50 \mathrm{~cm}$. «Window» consistía en llenar el espacio con tiras de aluminio de $30 \mathrm{~cm}$ de largo por $1,25 \mathrm{~cm}$ de ancho que reradiaban la onda emitida desde el radar en la misma longitud de onda. "Window» era más eficaz si la longitud de onda original era la mitad de larga que la tira de aluminio. Para una radiación en su banda de frecuencia, cada tira reflejaba el eco de forma equivalente a como lo haría la superficie de un bombardero pesado B-17 o B-24. Así, cuando se dejaban caer miles de tiras de aluminio de estas características desde un avión, alrededor de medio kilómetro antes de la llegada del escuadrón, el operador veía cómo su radar quedaba sumido en la más absoluta confusión, y con ello el armamento antiaéreo permanecía completamente cegado ${ }^{41}$. Las contramedidas eran muy eficaces. Se podría decir que Kuhn sirvió en uno de los laboratorios de guerra que ayudó a causar un mayor número de bajas tanto militares como civiles, al margen del «Proyecto Manhattan». Tanto antes como después del «Día D», los bombarderos aliados soltaron sobre el territorio europeo una enorme cantidad de bombas que contribuyeron al desgaste de la industria alemana, el ejército y la población civil ${ }^{42}$. Las contramedidas contribuyeron a que las campañas tuvieran menos bajas y más «éxitos». Un ejemplo fue la «Operación Gomorra», que arrasó Hamburgo. ${ }^{43}$ En Hamburgo, relata Freeman Dyson, las luces del fuego antiaéreo vagaban inseguras por el cielo mientras los bombarderos lograban que el aire «ardiese» a su paso ${ }^{44}$.

40 Cf. KUHN (s.f. $[a]$ ), p. 1; KUHN (s.f. [b]), Notes on Jamming Effectiveness Measurements. En TSKP, 1.8; KUHN, T.S. y SUTRO, P.J. (s.f.), Theory of Ship Echoes as Applied to Naval RCM Operations. En TSKP, 1.8.

41 KuHN (s.f. [c]), Use of «Window» in Aircraft of 9th Bombardment Division. En TSKP, 1.10; BROWN (1999), pp. 286 y 295-97; JONES, R.V. (1978), Most Secret War, Londres, Hamish Hamilton, pp. 287-99.

42 Cf. KeVles (1995), pp. 316-19.

43 Cf. BUdERI (1996), p. 192; JONES (1978) pp. 300-305.

44 Dyson, F. (1979), Disturbing the Universe, Nueva York, Basic Books, p. 20. 


\subsection{El periodo en Europa}

Kuhn trabajó en contramedidas en Harvard desde el principio del verano de 1943 hasta mayo de 1944. Después ocupó varios puestos sucesivos. En todos ellos, su trabajo fue, aunque algo variado, idénticamente restringido a este único campo. Ese mes salió para un segundo destino que él mismo solicitó: el American-British Laboratory que el Radio Research Laboratory tenía el campus del Telecommunications Research Establishment, en Great Malvern, Worcestershire, Gran Bretaña ${ }^{45}$. Durante los meses siguientes, Kuhn estaría enormemente cerca de la acción. Poco tiempo después, pasaría a la unidad de inteligencia técnica de la Fuerza Aérea Estratégica de Estados Unidos, en Bushy Park, Londres. Allí, entre otras tareas, adiestraba a los que interrogaban a los prisioneros de guerra, para extraer a estos últimos información acerca de los progresos del radar alemán, sus contramedidas y los nombres de los científicos alemanes ocupados en ello ${ }^{46}$. Pero Kuhn tampoco se estableció en Londres. Del 25 de agosto al 12 de septiembre de 1944 estuvo destinado en Francia. Kuhn se puso un uniforme de militar por primera vez en su vida, lo que evitaría una acusación de espionaje en caso de ser capturado. Su misión iba a ser examinar los emplazamientos de radar alemán que fueran dejados tras de sí a medida que Hitler se batía en retirada. Kuhn se dirigió a St. Jacques, al sur de Rennes, donde la Fuerza Aérea de Estados Unidos tenía situado un cuartel. Desde allí, Kuhn iría al propio Rennes, donde debía examinar un emplazamiento de radar de cierto calibre. Tras un cierto periplo de tintes algo novelescos, Kuhn acabaría en París a tiempo para asistir a la entrada triunfal de De Gaulle. A mitad de septiembre volvió a Londres, para regresar por segunda vez a Francia (y desde allí viajar a Holanda y Alemania) en ene-

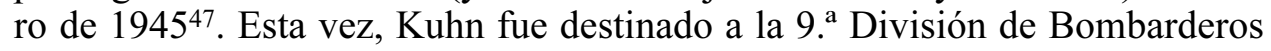
de la Fuerza Aérea de Estados Unidos, sita en Rheims ${ }^{48}$. La OSRD deseaba extender a esa división el uso de contramedidas (que ya habían dado muy buenos resultados en la 8. a División) y Kuhn era uno de los técnicos más adecuados para supervisar esa tarea ${ }^{49}$. En la 9. ${ }^{a}$ División, Kuhn tuvo que diseñar

45 Baltas et al. (2000), p. 269.

46 Cf. KUHN (1944a), Outline for Briefing P/W Interrogators. En TSKP, 1.8.

47 KUHN (1944b), [Diario de su estancia en Francia]. En TSKP, 1.10, pp. 1-9; Baltas et al. (2000), pp. 269-71.

48 Cf. la carta de John N. Dyer (Director del American-British Laboratory) al General Hoyt S. Vandenberg (9. ${ }^{\text { F.A. }}$ ), 17 de enero de 1945. En TSKP, 1.9.

49 Taylor, L.S. (1945), Assignment of Thomas S. Kuhn to Operational Research Section. En TSKP, 1.9. 
todo un programa de contramedidas adecuado a los modelos de bombardero que allí se utilizaban. Entre él y A.T. Goble, diseñaron prototipos de abertura para la expulsión de contramedidas. El trabajo se hacía difícil por las dificultades de abastecimiento y era engorroso debido a los numerosos informes que era necesario escribir acerca de la puesta en práctica del recurso ${ }^{50}$.

El 13 de mayo de 1945 este trabajo tocó a su fin. La guerra en Europa había terminado. No obstante, esta agotadora labor no hizo más que reanudarse en otro lugar. Kuhn empezó el mismo 13 de mayo a inspeccionar, uno por uno, los aparatos de radar que los alemanes se habían dejado tras de sí, así como a entrevistarse con científicos alemanes e interrogarlos sobre esta materia. El ajetreado itinerario de ese mes le llevó por diversas partes de Alemania: a Süchsteln (14 de mayo), al cuartel general del ejército; a Stade (16-17 de mayo), para la inspección del aeródromo; a Hamburgo (18-19 de mayo), para la inspección de los radares, las contramedidas y la defensa antiaérea, e interrogar a oficiales alemanes de radar sobre los mismos aspectos del radar alemán, así como sobre la efectividad de las contramedidas aliadas; a Pelzerhaken (20-21 de mayo), donde inspeccionó la principal estación experimental de radar de la marina alemana y se entrevistó con el personal de investigación; y a Bergedorf (22 de mayo), donde interrogó al Dr. Möller, director del Instituto Max Wien, sobre sus investigaciones teóricas de los tubos de vacío. Tras ello, el 24 de mayo regresó a París ${ }^{51}$.

Poco después, Kuhn volvió por fin a Harvard. Aún era posible que fuera enviado al Pacífico, en concreto a Japón, para tareas semejantes a las desempeñadas hasta el momento, pero el verano transcurrió sin noticias. En septiembre de 1945, Kuhn solicitó el ingreso en el programa de doctorado del Departamento de Física de Harvard. Pero su entrada en el Departamento ya estuvo marcada por una cierta falta de entusiasmo. El trabajo de física durante la guerra mostraba hasta qué punto ese ejercicio podía ser restrictivo, limitado e incluso monótono. Recordemos que su interés en la física había nacido, entre otras cosas, de su atracción por los problemas fundamentales, pero quizá la física no le permitiese acceder fácilmente a aquellos que le interesaban realmente. Siguiendo sus inclinaciones filosóficas de 1943, cultivadas aún más durante la guerra (cf. la sección siguiente), Kuhn pidió permiso para se-

50 Cf. las cartas de Thomas S. Kuhn a John N. Dyer del 28 de enero, el 17 de febrero, el 12 de marzo y el 2 de abril de 1945. En TSKP, 1.9. Cf. también KUHN (1945b), Desirability of a Carpet Program for Daylight Operations of Medium and Light Bombers. En TSKP 1.9.

51 Kunn, T.S. y LuSTED, L.B. (1945), Activities of Radar Investigator Attached to Navy Trip \#105. En TSKP, 1.10, pp. 1-2. 
guir unos cuantos cursos de doctorado del Departamento de Filosofía, algo claramente anómalo para un futuro físico ${ }^{52}$. Kuhn se estaba alejando de la física ortodoxa con pasos como éste, que no sería ni único ni el más crucial de los que diese en los años siguientes. Décadas después recordaba de este periodo que «con cada vez mayor frecuencia [...] me preguntaba si lo que yo realmente quería era una carrera de físico» ${ }^{53}$. La respuesta final a esa pregunta fue, como sabemos, un «no» rotundo. Su doctorado le permitió obtener un título y encontrar mientras tanto un modo de aprovechar sus conocimientos de física, pero ya fuera de la física. Por otro lado, el trabajo que llevó a cabo en esos años continuó siendo análogo a lo ya visto en el periodo de guerra, en lo referente a la limitación del punto de vista y al aumento de la especialización. $\mathrm{Si}$, en efecto, la ciencia normal de Kuhn se asienta parcialmente sobre su propia experiencia de la ciencia, esa base se adquiere sobre todo en los años y el trabajo que estamos examinando en esta sección y en la siguiente.

\section{KUHN Y LA FÍSICA DEL ESTADO SÓLIDO}

En su última entrevista en 1995, Arístides Baltas le preguntó a Kuhn si sus dudas sobre su dedicación a la física estaban relacionadas con el trabajo realizado durante la guerra. La respuesta de Kuhn indica que, más que dudas, en aquellos momentos tenía algunas certezas que ya no podía seguir eludiendo:

Yo había sido un «físico». Utilizo comillas ahora porque, en algún sentido, en vista de lo que había ocurrido, no había recibido la formación propia de un físico, pero había llegado a esto y yo lo empezaba a encontrar aburrido, el trabajo no me interesaba. [...] estaba empezando a dudar de si una carrera profesional como físico era lo que deseaba realmente — en particular en el ámbito de la física teórica—. Y creo que quizá haya sido entonces, aunque quizá fue más tarde, cuando empecé a plantearme la pregunta: ¿por qué quería ser un físico teórico? Las dudas no eran muy grandes aún, ni nada parecido, pero ya se habían planteado ${ }^{54}$.

Incluso, recordaba, la autoestima pareció fallarle: «[...] aunque continuaba yéndome lo suficientemente bien», decía Kuhn, «ya no era un niño prodigio, y no estaba claro que fuera lo suficientemente bueno... quiero decir para bri-

52 BALtAs et al. (2000), p. 273.

53 SigurdSSON (1990), p. 19; BALTAS et al. (2000), pp. 271-272.

54 BALTAS et al. (2000), p. 272; empleamos la traducción de A. Beltrán y J. Romo en Kunn, T.S. (2002), El camino desde la estructura, Barcelona, Paidós, p. 318. 
1lar realmente» ${ }^{55}$. Había un ingrediente añadido ya antes mencionado: la filosofía de la ciencia y, en concreto, algunos coqueteos con Kant y con Hume en relación con la función de los conceptos en la construcción de la teoría física, que habían nacido de la asignatura de Demos y, desde entonces, le habían ocupado sus escasos periodos de ocio. Durante la guerra, S. Freud, F. Nietzsche, W. James, P.W. Bridgman y B. Russell (junto a otras lecturas de divulgación científica de la mano de A.S. Eddington, R.A. Millikan, J.B.S. Haldane o M. Born) habían sido una vía de escape al trabajo de contramedidas ${ }^{56}$. Su vocación de físico estaba en el filo de la navaja y, ahora que la obligación para con el servicio de guerra había cesado (o estaba a punto de hacerlo), parecía el momento de replantearse seriamente la carrera profesional.

Pero Kuhn ya era físico; o "físico», con comillas, según él mismo quería matizar, una suerte de experto en ingeniería electrónica, más bien. Así que no iba a volver sobre sus pasos para empezar de nuevo toda su carrera académica como filósofo. El que Kuhn eligiese dedicar una parte de sus cursos de doctorado a la filosofía hacía ver que su dedicación a la física no iba a ser absoluta. De hecho, ni su tesis ni su director decían lo contrario, porque Kuhn trabajó en la reelaboración de una herramienta de cálculo para la física del estado sólido, no en un problema fundamental, y además no lo hizo con una de las «estrellas» del departamento, como podría haber sido Julian Schwinger ${ }^{57}$, sino con una antigua figura del panorama de la física teórica norteamericana: J.H. Van Vleck, su antiguo jefe en el Radio Research Laboratory, quien parecía conocer bien los quebraderos de cabeza de Kuhn y no le ponía obstácu$\operatorname{los}^{58}$. No sólo le propuso la investigación en sí, sino que parece haberle apoyado en un terreno que, Kuhn consideraba, era más psicológico que técnico ${ }^{59}$. La tesis con Van Vleck, más que un final triunfal, era una salida honrosa para este futuro ex-físico. Pero, ¿quién era, a fin de cuentas, Van Vleck?

55 BALTAS et al. (2000), p. 273; Kuhn (2002), p. 320.

56 Cf. MAYORAL (2009), §§2-3 y apéndice (p. 183).

57 Sobre Schwinger cf. SCHWEBER, S.S. (1994), QED and the Men Who Made It, Princeton, Princeton University Press, cap. 7.

58 Aunque sí parece que Kuhn siguió un curso con Schwinger en este periodo, como atestigua el escrito del primero: Aplication of Integral Iteration Methods to Nuclear Force Problems, 19 de enero de 1947, para Physics 47(a), de Schwinger. TSKP 1.5.

59 Cf. KuHn, T.S. (1948), The Cohesive Energy of Monovalent Metals as a Function of their Atomic Quantum Defects. Tesis Doctoral, Departamento de Física de la Universidad de Harvard, esp. p. vii. 


\subsection{Van Vleck}

Lo primero que hay que decir sobre Van Vleck es que no era en absoluto una opción equivocada. Más aún, una tesis doctoral bajo la tutela de Schwinger suponía (tal como escribía Philip Anderson en su autobiografía ${ }^{60}$ ) esperar, día sí, día no, en la puerta de su despacho, mientras que Van Vleck siempre estaba dispuesto a escuchar y reflexionar sobre los problemas de sus doctorandos (de hecho, el propio Anderson, cuyos objetivos como físico eran considerablemente más sólidos que los de Kuhn, «también» lo eligió como director de tesis). Ahora bien, trabajar con Van Vleck implicaba dedicarse a un terreno específico de problemas. ¿A qué se dedicaba Van Vleck fuera del servicio de guerra?

Van Vleck era hijo y nieto de matemáticos. Su abuelo y su padre habían ocupado sendas cátedras de matemáticas en la Wesleyan University. Van Vleck se doctoró en Harvard en 1922 bajo la dirección de Edwin C. Kemble (quien también sería un buen conocido de Kuhn). Dio clases en Harvard desde 1934, donde, diecisiete años después, ocuparía la prestigiosa Cátedra Hollis de Matemáticas y Filosofía Natural (la más antigua del país, fundada en 1727). Como físico teórico, su trabajo tuvo un enorme impacto en la física del estado sólido. Obtuvo el Premio Nobel junto a Neville Mott y el propio P.W. Anderson en 1977. Para llegar ahí, comenzó en la física atómica de la mano de Kemble, siendo uno de los primeros físicos norteamericanos netamente teóricos. En la línea de Kemble, aplicó la antigua teoría cuántica a los datos obtenidos de las líneas de absorción y emisión espectroscópicas, un trabajo del que salió un manual (Quantum Principles and Line Spectra) y varios artículos de gran interés. A partir de la segunda mitad de los años veinte se dedicó a estudiar la física del estado sólido mediante la nueva mecánica cuántica. En concreto, estudió las susceptibilidades eléctrica y magnética, que presentaban problemas nacidos de la aplicación de la antigua teoría cuántica al estudio de las propiedades de los sólidos. Su investigación dio como fruto una estructura general en términos cuánticos para las expresiones de las susceptibilidades magnéticas y eléctricas, que constituyó el aclamado The Theory of Electric and Magnetic Susceptibilities. El resto de su carrera discurrió ya por este terreno, que no sería física fundamental hasta los años setenta ${ }^{61}$.

60 En www.nobelprize.org/nobel_pzizes/physics/laureates/1977/anderson-autobio.html (consultado el 25 de febrero de 2011).

61 Sobre Van Vleck, cf. Mehra, J. y Rechenberg, H. (1982), The Historical Development of Quantum Theory, Nueva York, Springer, vol. 2.II, p. 418, nota 677; HodDESON, L., 
Una vez acordada su supervisión, el trabajo que Van Vleck encomendó a Kuhn fue la mejora del método de cálculo de la estructura de bandas de un metal. Ésta era una labor nada sencilla. Para comprender su alcance real conviene que partamos de, al menos, una descripción somera del estado de la cuestión ${ }^{62}$.

\subsection{El estado de la cuestión: Wigner y Seitz ${ }^{63}$}

A comienzos del siglo XX, la antigua teoría cuántica había ayudado a que las diversas teorías previas, no siempre compatibles, sobre las propiedades de los sólidos (i.e., estructura material, cohesión, plasticidad, conductividad, propiedades magnéticas, etc.) dispusiesen de un cuerpo teórico unificado. Por su parte, la cristalografía de rayos $\mathrm{X}$ había ayudado a obtener una perspectiva regular de la red cristalina. Pero la primera explicación cualitativa de las propiedades de los sólidos llegaría con la aplicación de la mecánica cuántica y, en concreto, de la estadística de Fermi-Dirac al estudio de los gases electrónicos, combinada con la teoría de bandas. Según esta última, la interacción entre los átomos en un sólido hace que los niveles electrónicos de aquéllos se desdoblen en tantos otros como átomos hay en el sólido; la proximidad de los niveles nos permite considerarlos una banda continua. La banda de los niveles superiores es más ancha y sus electrones se mueven casi libres por la red, con

Braun, E., Teichmann, J. y Weart, S. (eds.) (1992), Out of the Crystal Maze, Oxford, Oxford University Press, pp. 408-409. De su trabajo, cf. VAN VLECK, J.H. (1924a), The Absorption of Radiation by Multiply Periodic Orbits, and its Relations to the Correspondence Principle and the Rayleigh-Jeans Law. Part I. Some Extensions of the Correspondence Principle, Physical Review, 24, pp. 330-346; VAN VLECK, J.H. (1924b), The Absorption of Radiation by Multiply Periodic Orbits, and its Relations to the Correspondence Principle and the RayleighJeans Law. Part II. Calculations of Absorption by Multiply Periodic Orbits, Physical Review, 24, pp. 347-365; VAN Vleck, J.H. (1926), Quantum Principles and Line Spectra, Washington, National Research Council of the National academy of sciences, 54 (10); VAN VLECK, J.H. (1932), The Theory of Electric and Magnetic Susceptibilities, Oxford, Oxford University Press.

62 Para más información, cf. ECKERT, M. y Schubert, H. (1990), Crystals, Electrons, Transistors, Nueva York, American Institute of Physics, pp. 69-73 y 209-212; HodDESON, L. (1997), Solid State Science. En Krige, J. y Pestre, D. (eds.), Science in the Twentieth Century, Amsterdam, Harwood, pp. 585-598, esp. pp. 585-588; HODDESON et al. (eds.) (1992), pp. $15-27$ y $107-123$.

63 Agradezco a Javier García Sanz su colaboración específica en esta breve sección y en la siguiente. 
lo que podemos hablar de un gas de electrones libres. La estadística de FermiDirac impone que en el estado fundamental el gas está degenerado, así que los niveles de menor energía dentro de la banda se ocupan hasta alcanzar un nivel máximo, el «nivel de Fermi». Mientras que en los sólidos aislantes este nivel satura toda la banda, en los metales hay menos electrones libres de los que la banda es capaz de acoger, así que hay niveles no ocupados por encima del de Fermi. Por el principio de exclusión de Pauli, al aplicar una fuente de energía externa sólo los electrones que ocupan un nivel energético próximo al de Fermi pasan a otro superior no ocupado, con lo que, en condiciones normales de temperatura, sólo alrededor del $1 \%$ de los electrones de la banda contribuye a la conductividad térmica y eléctrica y al calor específico. La explicación así obtenida es útil desde un punto de vista cualitativo, pero no cuantitativo. Este segundo apareció con el trabajo de Eugene Wigner y su doctorando Frederick Seitz (1933), quienes aportaron el primer método de cálculo de las energías de cohesión aplicado a un metal alcalino, el sodio ${ }^{64}$. Es importante ver brevemente cómo era este método, ya que el trabajo de Kuhn y Van Vleck parte directamente de él.

En su artículo de 1933, Wigner y Seitz diferenciaban celdas en la red metálica a partir los planos cristalográficos de simetría que bisecan las líneas que unen a cada átomo con sus vecinos inmediatos. Con ello, estudiaban la energía del electrón y su función de onda como si formasen parte de un átomo libre, sólo que con las características propias de un átomo en una red cristalina. Así, mientras que la probabilidad de presencia de un electrón en un átomo libre (expresada en términos del cuadrado de la función de onda del electrón) tiende a cero a medida que aumenta la distancia al núcleo atómico, la del electrón de la red metálica en la misma situación alcanza valores finitos. El electrón de valencia presenta un máximo de energía a la mitad de la distancia interatómica y su función de onda es continua de forma periódica por toda la red cristalina. Su movimiento depende del átomo vecino, cuya presencia reduce aún más el valor de su energía potencial. Al tiempo, su energía cinética es menor que en el caso del átomo libre. Sobre esta base, Wigner y Seitz hallaban la función de onda real del electrón en la red cristalina mediante una aproximación en la que hacían periódicamente continua la función de onda en el caso atómico. La clave era calcular la energía del electrón libre en su estado fundamental, algo que lograban resolviendo numéricamente la correspondiente ecuación de Schrödinger, tomando como condiciones de contorno que

64 Wigner, E. y Seitz, F. (1933), On the Constitution of Metallic Sodium, Physical Review, 43, pp. 804-10. 
la derivada de la función de onda se anule en los límites de la celda (los planos cristalográficos de simetría que bisecan las líneas que unen átomos vecinos). Una vez obtenida la función de onda, podían proporcionar resultados numéricos de algunas de las propiedades químicas del sodio metálico, como la constante de equilibrio de la red de sodio o la energía de enlace ${ }^{65}$.

Con este trabajo y su ampliación ${ }^{66}$, Wigner y Seitz aportaron los primeros cálculos de la estructura de bandas de un metal real. Había, no obstante, mejoras que hacer en él, fundamentalmente matemáticas, que consistían en lograr superar los tediosos cálculos que el método requería ${ }^{67}$. El trabajo de Kuhn y Van Vleck se encontraba en esta línea.

\subsection{Mejoras normales: Kuhn y Van Vleck}

La investigación de Kuhn con Van Vleck permitía esquivar algunas de las dificultades matemáticas de la aproximación mediante celdas que acabamos de ver. No se apartaba lo más mínimo de los objetivos ni del planteamiento de Wigner y Seitz, no ofrecía otro modo de cálculo de parámetros alternativo al de estos, ni se salía de su método de celdas. Esto recuerda mucho a ciertas afirmaciones que Kuhn escribiría más tarde en Structure:

Pocas personas que no sean de hecho científicos practicantes de una ciencia madura se darán cuenta de hasta qué punto un paradigma deja sin hacer una gran cantidad de trabajo de retoque [...], o lo fascinante que puede ser la realización de este trabajo. Hay que comprender estos aspectos. Las operaciones de retoque ocupan a la mayoría de los científicos a lo largo de sus carreras. Constituyen lo que llamo aquí ciencia normal. Si se examina detenidamente, sea históricamente o en el laboratorio contemporáneo, dicha empresa parece ser un intento de meter a la fuerza a la naturaleza en los compartimentos prefabricados y relativamente inflexibles suministrados por el paradigma. [... No] entra normalmente entre los objetivos de los científicos inventar teorías nuevas [...]. Por el contrario, la investigación en ciencia normal se orienta a la articulación de los fenómenos y teorías ya suministrados por el paradigma.

65 Cf. Hoddeson, L. y Daitch, V. (2002), True Genius: The Life and Science of John Bardeen, Washington, Joseph Henry Press, cap. 4, esp. pp. 60 y ss., para una perspectiva semejante a ésta pero desde el punto de vista del físico John Bardeen.

66 Wigner, E. y Seitz, F. (1934), On the Constitution of Metallic Sodium, II, Physical Review, 46, pp. 509-24.

67 Cf. SEITZ, F. (1981), Entrevista con Lillian Hoddeson, 26 de enero. Niels Bohr Library, American Institute of Physics, Nueva York. 
Quizá sean defectos. Las áreas investigadas por la ciencia normal son minúsculas, por supuesto, pues la empresa que ahora se discute posee una visión drásticamente reducida. Sin embargo, tales restricciones surgidas de la confianza en un paradigma resultan ser esenciales para el desarrollo de una ciencia ${ }^{68}$.

Dejemos de lado la perspectiva claramente interna al trabajo científiconormal que Kuhn adopta en el pasaje seleccionado para describir ese modo de investigación (atiéndase sobre todo a las primeras frases del extracto). Lo que vamos a ver a continuación encaja en la idea del fascinante (aunque correspondiente a un área de investigación minúscula) «trabajo de retoque», que no requiere «inventar teorías nuevas» sino «articular otras ya suministradas por el paradigma». En The Essential Tension, Kuhn realiza una breve, pero sugerente descripción de este tipo de investigación científica: «En la ciencia pura o básica [...] los problemas característicos son casi siempre repeticiones, con modificaciones menores, de problemas antes tratados y ya parcialmente resueltos ${ }^{69}$. El trabajo de Kuhn y Van Vleck se amolda bastante bien a esta perspectiva, como veremos a continuación.

En su tesis, Kuhn trabajó en dos métodos: el llamado Function Matching Method, desarrollado en colaboración con Van Vleck, ${ }^{70} \mathrm{y}$ una aplicación propia del método WKB que el japonés Isao Imai había desarrollado en Tokio ${ }^{71}$. Con el primer método, Kuhn y Van Vleck mejoraban ostensiblemente las aproximaciones de Wigner y Seitz para las constantes de red, las energías de cohesión y la compresibilidad (cf. nuestro Apéndice, más abajo) ${ }^{72}$. La varia-

68 KuHN (1962), p. 24. Citamos según la nueva traducción reciente de Structure: KuHN (2006), La estructura de las revoluciones científicas, trad. e introd. de C. Solís, Méjico, FCE, pp. 89-90.

69 KuHN (1977), p. 233. Tanto el pasaje del que hemos obtenido este extracto, como Structure, §III, ofrecen ricas descripciones de los tipos de trabajo que pueden ser clasificados como «ciencia normal».

70 KUHN (1948), caps. II-IV.

71 Kuhn (1948), caps. V-VI. Cf. IMAI, I. (1948), On a Refinement of the W.K.B. Method, Physical Review, 74, p. 113. Sobre el método WKB en sí, cf. los artículos de los científicos cuyas iniciales dan nombre al método: WeNZEL, G. (1926), Eine Verallgemeinerung der Quantenbedingungen fur die Zwecke der Weellenmechanik, Zeitschrift für Physik, 39; Kramers, H. (1926), Wellenmechanik und Halbzahlige Quantisierung,. Zeitschrift für Physik, 39 y Brillouin, L. (1926), Notes on Undulatory Mechanics, Journal de physique théorique et appliquée, 7. Para un estudio filosófico reciente del método WKB, cf. BATTERMAN, R. (2002), The Devil in the Details: Asymptotic Reasoning in Explanation, Reduction and Emergence, Oxford, Oxford University Press, §7.2.

72 Kuhn, T.S. y VAn Vleck, J.H. (1950), A Simplified Method of Computing the Cohesive Energies of Monovalent Metals,. Physical Review, 79, pp. 382-88. 
ción introducida consistía en evitar el cálculo del potencial del campo central del átomo, algo necesario para obtener la función de onda del electrón. Para ello, Kuhn y Van Vleck asumían que la constante de red, la energía de cohesión y la compresibilidad dependían de las funciones de onda en una región exterior al corazón del átomo. La derivada logarítmica de estas funciones de onda debe satisfacer una condición que sólo depende de la energía de la función de onda (ya no del potencial del campo) y éste se puede obtener de los valores de laboratorio de los primeros niveles de energía del átomo libre ${ }^{73}$. Por otro lado, las soluciones de la ecuación de onda correspondiente son idénticas a una determinada combinación lineal de dos funciones conocidas que, a su vez, son solución de la ecuación hipergeométrica confluyente resuelta por E.T. Whittaker ${ }^{74}$. Ahora bien, para aplicar este método había que conocer los valores de las funciones-solución de Whittaker dentro de un ámbito físicamente significativo de los mismos que superaba a aquél para el que ya existían cálculos realizados. Puesto que su cálculo era virtualmente impracticable, Kuhn desarrolló una solución general de la ecuación diferencial mucho más manejable que la de Whittaker. Kuhn expuso sus resultados en un artículo que publicó en el Quarterly of Applied Mathematics ${ }^{75}$. Por fecha, ésta sería su última publicación científica ${ }^{76}$.

En cuanto al segundo método, el WKB permitía obtener una solución para la ecuación de onda sin emplear la combinación lineal de las soluciones de Whittaker. El método WKB proporciona una solución asintótica de una ecuación diferencial de segundo orden. En la primera formulación del método, la función que proporciona una solución para la ecuación de onda se comporta de manera diferente a ambos lados del punto de retorno clásico, bien como una función exponencial (en la región clásicamente prohibida), bien como un

73 KUHN y VAN VLeCK (1950), p. 383.

74 Kuhn y Van Vleck (1950), p. 385; Whittaker, E.T. y Watson, G.N. (1927), A Course of Modern Analysis, Cambridge, Cambridge University Press, pp. 337 y ss.

75 KuHN (1951), A Convenient General Solution of the Confluent Hypergeometric Equation, Analytic and Numerical Development, Quarterly of Applied Mathematics, 9, pp. 1-16. Este desarrollo general de Kuhn ya había sido explorado antes por G.H. Wannier y por R. Jastrow en 1943 y 1948, respectivamente.

76 En sus archivos del MIT hay constancia de que Kuhn preparó dos presentaciones sobre su trabajo de doctorado para sendas reuniones científicas celebradas en mayo y junio de 1951 . La primera se titulaba Atomic Quantum Defects Applied to the Solid State, y en ella ya reflejaba (cf. su p. 1) los artículos publicados que mencionamos aquí (KUHN y VAN VLECK [1950], KuHN (1950), (1951)]. La segunda era Computing Metallic Cohesive Energy without the Use of Potential Functions. Ambas se encuentran en TSKP 12.33. 
seno (en la región clásicamente permitida). La región en que las funciones de onda son físicamente significativas se encuentra cerca de ese punto, pero sólo R.E. Langer en los años treinta había logrado conectar ambas regiones ${ }^{77}$. Isao Imai mejoró en 1948 la aproximación de Langer y Kuhn empleó ese trabajo en su tercer artículo para Physical Review para obtener resultados numéricos semejantes a los ya conseguidos mediante el método Kuhn-Van Vleck ${ }^{78}$. Con este segundo método, sin embargo, se evitaba la insegura extrapolación de la derivada logarítmica en función de valores dados de energía empleada en el método anterior. Para ello, Kuhn se apoyaba en los valores experimentales, más fiables, del conocido como «defecto cuántico», necesarios para la aproximación en la zona exterior, próxima al punto de retorno. El «defecto cuántico» es una medida de la desviación de la órbita del electrón de valencia del átomo libre con respecto a su correspondiente órbita hidrogenoide. Expresado de otro modo, el «defecto cuántico» indica la medida de penetración de la órbita estacionaria en la capa formada por los electrones que rodean al núcleo $^{79}$. Es una medida experimental mediante la cual Kuhn calculaba la constante de fase necesaria para obtener las funciones de onda ${ }^{80}$.

Durante la década de 1950, el trabajo de Kuhn con Van Vleck se incluyó entre las mejoras y desarrollos del método de celdas ${ }^{81}$. Fue desarrollado por Harvey Brooks e incluso llegó a ser objeto de una tesis doctoral en Harvard, la de F.S. Ham, todo ello hasta mediados de los cincuenta ${ }^{82}$. Después su importancia ha sido meramente teórica, ya que los métodos empleados actualmente difieren mucho del esquema Wigner-Seitz ${ }^{83}$. Se trata, por lo tanto, de un trabajo que contribuye a aproximar de manera más precisa la teoría al experimento, al tiempo que logra hacer algo más manejable (e incluso fiable) la primera. Emplea nuevos recursos disponibles para aumentar las virtudes me-

77 IMAI (1948), p. 113.

78 Kunn, T.S. (1950), An Application of the W.K.B. method to the Cohesive Energy of Monovalent Metals, Physical Review, 79, pp. 515-519.

79 Cf. D. HAAR, Ter (1967), The Old Quantum Theory, Oxford, Pergamon, p. 54; VAN VLECK (1932), pp. 215-20.

80 KuHN (1950), p. 517.

81 Reitz, J.R. (1955), Methods of the One-Electron Theory of Solids, Solid State Physics, 1, pp. 2-96, esp. pp. 15-17.

82 Brooks, H. (1953), Cohesive Energy of Alkali Metals, Physical Review, 91, pp. 10271028; Ham, F.S. (1955), The Quantum Defect Method, Solid State Physics, 1, pp. 127-92.

83 Cf. Callaway, J. (1991), Quantum Theory of the Solid State, San Diego, Academic Press, p. 72; Haug, A. (1975), Theoretical Solid State Physics, Trad. de H.S.H. Massey, Oxford, Pergamon, vol. 2, pp. 82 y 236, nota. 
todológicas de una teoría ya en uso. No es un trabajo de crítica ni una investigación pionera, aunque requiera ingenio y habilidad. Es, como hemos indicado al principio de este apartado, el tipo de mejora propia de la ciencia normal que Kuhn retrató en su trabajo filosófico posterior.

\section{CONCLUSIÓN}

La investigación física de Kuhn con Van Vleck está altamente especializada. Resuelve problemas que contribuyen a la mejora de un método científico, a su aproximación a la observación, y a hacer más fácil su aplicación. Requiere conocimientos muy especializados obtenidos de una educación universitaria principalmente consistente en saber cómo resolver problemas cada vez más especializados en matemáticas y física. En esta educación hay poco tiempo, dentro de la adquisición de dicha maestría, para la discusión crítica y la consideración de alternativas, como tampoco lo hay en el ejercicio profesional posterior (y no digamos ya en el contexto, un tanto particular, de la investigación con fines militares inmediatos). La crítica y la consideración de alternativas quedan fuera de tales contextos, al margen de estas disciplinas.

Ésta era la experiencia de Kuhn con la física en 1951. Aunque Structure apareció una década después, entre los componentes con los que Kuhn confecciona su idea ciencia normal se encuentran los que acabamos de mencionar en el párrafo anterior. Teniendo esto en cuenta, junto con la afirmación ya vista del propio Kuhn de que algunas de sus ideas provienen de su experiencia en la investigación científica, podemos decir que uno de los aspectos más novedosos de Structure se originó, con un alto grado de probabilidad, en su experiencia personal con la física del siglo XX.

Esto contribuye a generar dudas acerca de la generalidad del punto de vista kuhniano. ¿No será la perspectiva kuhniana una imagen demasiado coyuntural de la ciencia? ¿Es realmente extrapolable a todo periodo de la ciencia? E incluso aunque admitiésemos que hay dos tipos opuestos de práctica científica, catalogables al modo kuhniano como «ciencia normal» y «ciencia extraordinaria», ¿es la imagen que Kuhn ofrece de ellos lo suficientemente completa y fiable? Al principio de este artículo hemos ofrecido una voz discordante, la de M. Beller, que responde afirmativamente a la primera cuestión, mientras que ofrece un «no» para las dos segundas. Para Beller, Kuhn cubre la ciencia normal con un manto de dogmatismo que es producto de su propia experiencia personal de la ciencia. Esta experiencia es limitada; está circunscrita a una fase, la del científico novel, donde hay menos lugar para la 
duda y la crítica que en la etapa del científico maduro. Beller se encuentra, por lo tanto, entre quienes consideran que la ciencia normal de Kuhn debe demasiado al propio Kuhn (a su perspectiva subjetiva). Discutir esta postura es sin duda interesante y probablemente ofrezca nuevas perspectivas para nuestra comprensión de la ciencia. En este artículo sólo hemos intentado ofrecer una base para iniciar esa discusión. Esperamos que futuros estudios contribuyan a dar el paso siguiente.

7. Apéndice: Comparación de Resultados de los trabajos De Wigner-Seitz Y KUHN-VAN VLECK ${ }^{84}$

\begin{tabular}{lccc}
\hline & Wigner-Seitz & Kuhn-Van Vleck & V. experimental \\
\hline $\begin{array}{l}\text { Constante de red } \\
(\AA)\end{array}$ & 4,74 & 4,14 & 4,25 \\
$\begin{array}{l}\text { Energía de cohesión } \\
(\text { Kcal/mol })\end{array}$ & 23,2 & 25,9 & 26 \\
$\begin{array}{l}\text { Compresibilidad } \\
\left(\mathrm{cm}^{2} / \text { dina }\right)\end{array}$ & --- & $13 \cdot 10^{-2}$ & $12,3 \cdot 10^{-2}$ \\
\hline
\end{tabular}

Recibido: 12 de marzo de 2009.

Aceptado: 13 de diciembre de 2010.

84 Fuente: KUHN y VAN VLECK (1950), p. 386. Los valores son para el sodio. 